\title{
Ultrasound Promoted Stereoselective Synthesis of 2,3-Dihydrobenzofuran Appended Chalcones at Ambient Temperature
}

\author{
Vishnu A. Adole* ${ }^{*} \$$, Bapu S. Jagdale, Thansing B. Pawar and Abhishek A. Sagane \\ Research Centre in Chemistry, Mahatma Gandhi Vidyamandir's Loknete Vyankatrao Hiray Arts, Science and Commerce College Panchavati, \\ Nasik-422003, India (Affiliated to Savitribai Phule Pune University, Pune)
}

Received 22 January 2019, revised 14 January 2020, accepted 21 January 2020.

\begin{abstract}
In the present investigation, an ultrasound promoted the synthesis of a series of (E)-3-(2,3-dihydrobenzofuran 5-yl)-1-(aryl)prop2-en-1-one derivatives from 2,3-dihydrobenzofuran-5-carbaldehyde and various aromatic ketones under clean conditions. The application of ultrasound irradiation in organic reactions is one of the incredible tools of green chemistry as reactions can be carried out rapidly under neat conditions. A library of a novel (E)-3-(2,3-dihydrobenzofuran-5-yl)-1-(aryl)prop-2-en-1-one chalcone derivatives were synthesized in good to excellent yield under ultrasonic irradiation. The structures of all synthesized chalcone derivatives synthesized in this study have been established by using FT-IR, ${ }^{1} \mathrm{H} N M R,{ }^{13} \mathrm{C}$ NMR, and HRMS techniques. The stereochemistry around $\mathrm{C}=\mathrm{C}$ in the chalcones was shown to be trans by ${ }^{1} \mathrm{H} N M R\left(J_{\mathrm{ab}}=15.5 \mathrm{~Hz}\right)$. The benefits of the present synthesis include mild reaction conditions, high yield, purification by non-chromatographic strategy and short reaction times, demonstrating the significance of this protocol in terms of waste reduction and energy efficiency.
\end{abstract}

KEYWORDS

2,3-Dihydrobenzofuran-5-carbaldehyde, ultrasound irradiation, green chemistry, trans chalcone.

\section{Introduction}

2,3-dihydrobenzofuran-5-carbaldehyde is a fused organic molecule wherein benzene is fused with a dihydrofuran ring. It has been used for the synthesis of a privileged scaffold 1,2,6,7-tetrahydro-8H-indeno[5,4- $b$ ]furan-8-one (used for the synthesis of ramelteon). The dihydrofuran ring has been identified as a necessary pharmacophore by which ramelteon works as a sleep agent in people having sleep-onset insomnia. ${ }^{1,2}$ Noteworthy examples of synthetic intermediates and drugs containing dihydrobenzofuran (Fig. 1) are 1,2,6,7-tetrahydro$8 \mathrm{H}$-indeno[5,4- $b$ ] furan-8-one (1), ramelteon (2), tasimelteon (3), 1-(2,3-dihydrobenzofuran-5-yl)propan-2-amine (4), 1-(2,3-dihydrobenzofuran-5-yl)-N-methylpropan-2-amine (5) and $((1 R, 2 R)-2-(2,3-d i h y d r o b e n z o f u r a n-4-y l) c y c l o p r o p y l)$ methanol (6). The presence of the dihydrofuran ring in 2,3-dihydrobenzofuran-5-carbaldehyde attracted us to synthesize a new series of chalcones with potential medicinal properties.

Chalcone is a common and simple scaffold found in many naturally occurring compounds showing excellent biological activities. ${ }^{3}$ Chalcones are a notable class of compounds, they also act as vital intermediates for the development of a variety of biologically potent heterocyclic compounds. ${ }^{4-6}$ Chalcones display a wide scope of biological properties, most likely because of their small structures and Michael acceptor features, which make them tolerant to various biomolecules and enabling them to react or interact with them. The Michael acceptor site can be tuned to augment the biological potential of chalcones. ${ }^{7}$ Chalcones containing a heterocyclic moiety display excellent biological profiles, including antiproliferative and anticancer, ${ }^{8-13}$ antioxidant, ${ }^{14,15}$ antiviral, ${ }^{16-19}$ anti-tubercular, ${ }^{20-22}$ antimicrobial, ${ }^{23-26}$ anti-Alzheimer, ${ }^{27}$ and antihypertensive properties. ${ }^{28}$

* To whom correspondence should be addressed.

E-mail: vishnuadole86@gmail.com
Some naturally isolated chalcones are explored as potent anticancer, anti-HIV, antioxidant, antimicrobial and antiinflammatory agents. ${ }^{29-34}$ The structural modification of aryl rings, supplanting of aryl rings and molecular hybridization through conjugation with pharmacologically active platforms for the improvement of anticancer properties have empowered advancement of new chalcones with potential therapeutic adequacy in diverse pharmacological applications. On these grounds, chalcones are considered important structures in medicinal chemistry.

The literature shows that different strategies have been used for the synthesis of chalcones. ${ }^{35}$ A large portion of reported strategies either require harsh conditions or prolonged reaction times. ${ }^{36}$ Organic syntheses utilizing the principles of green chemistry is the need of the present as well as the future. Numerous green chemistry oriented organic synthesis methods have been developed in order to synthesize a wide range of compounds with less environmental hazards. ${ }^{37-39}$ A standout amongst the most productive strategies as far as reaction time and yield, is ultrasound irradiation. ${ }^{40}$ Organic synthesis based on ultrasound irradiation has attracted the attention of chemists in the last two decades. In this regard, sonochemistry has been rapidly advanced in recent years and it is considered to be an important tool for green chemistry in terms of waste minimization and energy conservation. Ultrasound can adjust the selectivity performance of the reaction and enhances the rate of reaction; hence it has been employed more frequently for organic transformations. Under ultrasound irradiation, the reaction time diminishes considerably, the yields are higher, and the reaction conditions are benign. In continuation of our interest in the development of mild, operationally simple and environment-friendly protocols for the synthesis of pharmacologically important compounds, we envisaged access to the new 
<smiles>O=C1CCc2ccc3c(c21)CCO3</smiles>

1<smiles>CC(N)Cc1ccc2c(c1)CCO2</smiles>
4<smiles>CCC(=O)NCCC1CCc2ccc3c(c21)CCO3</smiles>

2<smiles>CNC(C)Cc1ccc2c(c1)CCO2</smiles>

5<smiles>CCC(=O)NC[C@@H]1C[C@@H]1c1cccc2c1CCO2</smiles>

3

\section{6}

Figure 1 Some biologically important intermediates and medicinal agents are containing a dihydrobenzofuran ring.

series of (E)-3-(2,3-dihydrobenzofuran-5-yl)-1-(aryl)prop2-en-1-one derivatives (9a-9n) of biological importance. Herein, we report the rapid and facile synthesis of (E)-3-(2,3-dihydrobenzofuran-5-yl)-1-(aryl)prop-2-en-1-one derivatives by using 2,3-dihydrobenzofuran-5-carbaldehyde and various aromatic ketones under ultrasonic irradiation. To the best of our knowledge, this is the first report on the synthesis of $(E)-3-(2,3-$ dihydrobenzofuran-5-yl)-1-(aryl)prop-2-en-1-one derivatives under ultrasonic irradiation. The utility and applicability of this green protocol are enhanced by its simple work-up procedure, rendering it readily adaptable for use in automated synthesis. The overall reaction is depicted in Scheme 1.

\section{Experimental}

\subsection{General Remarks}

2,3-Dihydrobenzofuran-5-carbaldehyde was purchased from MS Life Sciences, Hyderabad, India. Other chemicals (Make: Sigma-Aldrich, Merck, and Avra synthesis) with high purity were purchased from local distributors. All the chemicals were used as received without any further purification. Melting points were determined in open capillaries and are uncorrected. ${ }^{1} \mathrm{H}$ NMR and ${ }^{13} \mathrm{C}$ NMR spectra were recorded with a Bruker Avance III HD NMR $500 \mathrm{MHz}$ using $\mathrm{CDCl}_{3}$ as a solvent, FT-IR spectra were obtained with potassium bromide pellets and HRMS were recorded on a Bruker Impact HRMS with ESI as a source. All reactions reported in this study were monitored using thin-layer chromatography using aluminium sheets coated with silica gel 60 F254 (Merck).

2.2. General Procedure for the Synthesis of $(E)-3-(2,3-$ dihydrobenzofuran-5-yl)-1-(aryl)prop-2-en-1-one Derivatives

2,3-Dihydrobenzofuran-5-carbaldehyde (1 mmol), substituted acetophenones (1 mmol), absolute ethanol $(5 \mathrm{~mL})$, and $40 \%$ sodium hydroxide $(20 \mathrm{mmol}$ diluted up to $2 \mathrm{~mL}$ ) were added in a Pyrex flask $(25 \mathrm{~mL})$. The alkaline mixture was then exposed to ultrasound irradiation at room temperature for the period as indicated in Table 5 . The reaction was monitored by thin-layer chromatography (eluent 20:80: ethyl acetate: hexane). The reaction mixture was quenched by pouring on ice. After this, the resulting mixture was acidified by using dilute $\mathrm{HCl}$ and the resulting precipitate was then filtered and dried to give the desired product. The obtained product was recrystallized from hot ethanol. The synthesized pure products were characterized by FT-IR, ${ }^{1} \mathrm{HNMR},{ }^{13} \mathrm{C}$ NMR and HRMS analytical methods.

\section{Results and Discussion}

A broad range of chalcone analogs can conveniently be prepared from existing readily available substituted acetophenones and 2,3-dihydrobenzofuran-5-carbaldehyde. The synthesis of chalcones was accomplished by means of the ClaisenSchmidt reaction between 2,3-dihydrobenzofuran-5-carbaldehyde and various aromatic ketones. This scheme was envisaged to be highly advantageous due to the easy availability of a diverse range of aromatic ketones. The Claisen-Schmidt condensation between 2,3-dihydrobenzofuran-5-carbaldehyde (7) and acetophenone (8a) was selected as a model reaction for the optimization of reaction conditions for the synthesis of chalcones. The choice of base for the Claisen-Schmidt reaction is important to the success of this protocol. In order to explore the effect of different bases on the model reaction, the investigation was initiated with the selection of various bases. The easily available and common bases such as $\mathrm{NaOH}, \mathrm{KOH}, \mathrm{K}_{2} \mathrm{CO}_{3}, \mathrm{Na}_{2} \mathrm{CO}_{3}$, $\mathrm{NaHCO}_{3}, \mathrm{NaOMe}$, piperidine, pyrrolidine, $\mathrm{Li}(\mathrm{OH}) \cdot \mathrm{H}_{2} \mathrm{O}, \mathrm{NEt}_{3}$ and pyridine were screened for a model reaction (Table 1 ). The results obtained for a large portion of bases indicate the yield of the product 9a was not very satisfactory and they require a longer time for the completion of a reaction. It was revealed that the $\mathrm{NaOH}$ is a better choice as it furnished a maximum yield of 9a within shorter reaction time. To check the precise amount of $\mathrm{NaOH}$, we varied the amount of $\mathrm{NaOH}$ from $5 \mathrm{mmol}$ to $50 \mathrm{mmol}$ (Table 2). When the amount $\mathrm{NaOH}$ was increased from $5 \mathrm{mmol}$ to $20 \mathrm{mmol}$, the yield of $9 \mathrm{a}$ was found to increase with an overall reduction in completion time. However, the yield of 9 a dimin-<smiles>O=Cc1ccc2c(c1)CCO2</smiles><smiles>[R]c1ccc(C(C)=O)cc1</smiles>

8a to 8n

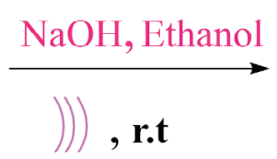

Synthesis of (E)-3-(2,3-dihydrobenzofuran-5-yl)-1-(aryl)prop-2-en-1-one derivatives under ultrasonic irradiation. 
Table 1 Effect of various bases on the synthesis of (E)-3-(2,3-dihydrobenzofuran-5-yl)-1-phenylprop-2-en-1-one (9a). ${ }^{a}$

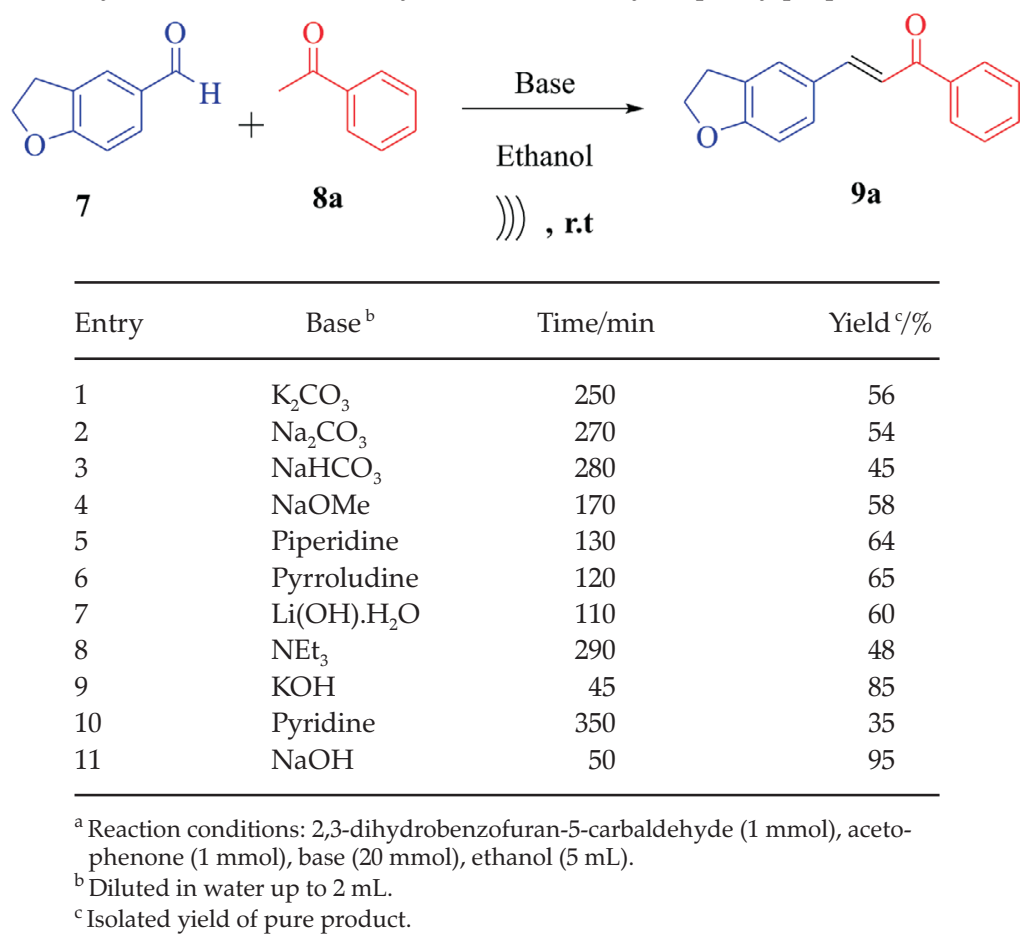

ished when the amount of $\mathrm{NaOH}$ was increased from $20 \mathrm{mmol}$ to $50 \mathrm{mmol}$. We found that $20 \mathrm{mmol}$ of sodium hydroxide was sufficient to ensure the reaction proceeded successfully. At the beginning of the selection of the best solvent, we applied the model reaction under solvent-free conditions and in the presence of various solvents (Table 3). The solvent-free reaction furnished the desired chalcone (9a) in very poor yield (entry $\mathbf{1}$ ). By contrast, the model reaction in other solvents afforded the desired chalcone in a satisfactory amount. A polar protic solvent such as ethanol and methanol were observed to be the most adequate as they yielded the chalcone in excellent yield. The formation of desired chalcone in the presence of polar aprotic solvents $\left(\mathrm{CH}_{3} \mathrm{CN}, \mathrm{DMF}, \mathrm{DMSO}, \mathrm{THF}\right)$ was more worthwhile in comparison to less polar and non-polar solvents $\left(\mathrm{CH}_{2} \mathrm{Cl}_{2} \mathrm{CHCl}_{3}\right.$ $\mathrm{PhCH}_{3}, \mathrm{CCl}_{4}$ ), yet not as efficient as in the presence of ethanol and methanol. The hydrogen bond-forming ability of ethanol and methanol makes them superior over other solvents. Among all the employed solvents, ethanol was found to be the most efficient in terms of reaction time and yield and was utilized for further investigations.

After this, traditional methods which are often used to achieve the desired goal were considered. Two traditional stirring and reflux methods were employed to compare with the expeditious ultrasound technique. The data obtained using these conventional methods suggest that ultrasonication is very much better in terms of yield and time (Table 4). These reactions require

Table 2 Optimization of a base for the synthesis of $9 a^{a}$

\begin{tabular}{lllll} 
& & & \\
\hline & & & & \\
Entry & \multirow{2}{*}{$\mathrm{NaOH}^{\mathrm{b}} / \mathrm{mmol}$} & $\mathrm{Time} / \mathrm{min}$ & Solvent & Yield $\mathrm{c} \%$ \\
\hline 1 & 5 & 320 & Ethanol & 20 \\
2 & 10 & 240 & Ethanol & 50 \\
3 & 15 & 100 & Ethanol & 58 \\
4 & 20 & 50 & Ethanol & 95 \\
5 & 25 & 45 & Ethanol & 94 \\
6 & 30 & 42 & Ethanol & 90 \\
7 & 35 & 40 & Ethanol & 88 \\
8 & 40 & 40 & Ethanol & 87 \\
9 & 45 & 38 & Ethanol & 80 \\
10 & 50 & 35 & Ethanol & 78 \\
\hline
\end{tabular}

a Reaction conditions: 2,3-dihydrobenzofuran-5-carbaldehyde (1 mmol), acetophenone $(1 \mathrm{mmol})$, ethanol $(5 \mathrm{~mL})$

${ }^{\mathrm{b}}$ Diluted in water up to $2 \mathrm{~mL}$ (except for $50 \mathrm{mmol}$ ).

${ }^{\mathrm{c}}$ Isolated yield of pure product. 
Table 3 Comparative analysis of various solvents for the synthesis of $9 a^{a}{ }^{a}$

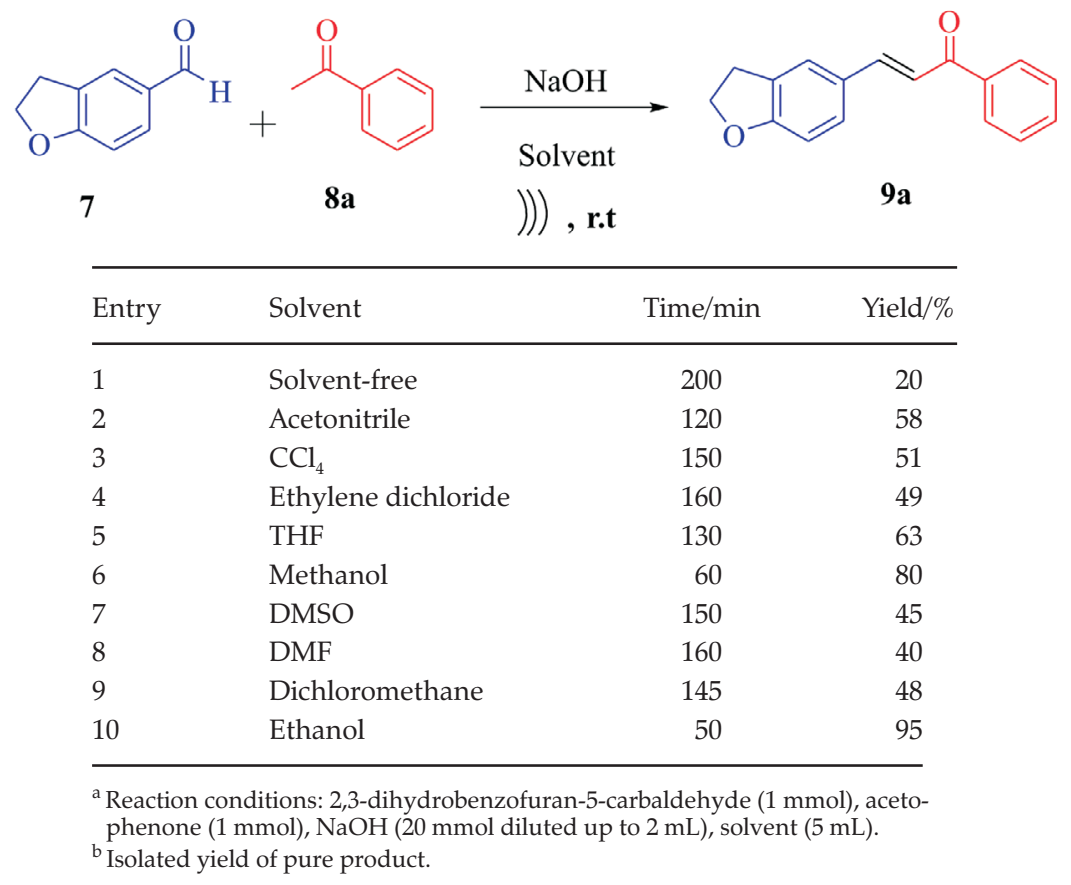

longer reaction times with lower yields, as compared to similar reactions under ultrasound irradiation. Following the establishment of optimal conditions to evaluate the scope and limitations of this green strategy, the present protocol was extended to a broader range of aromatic ketones and heterocyclic ketones. Results of the obtained yields tabulated in Table 5 indicate that an array of functionality was tolerated under optimized reaction conditions. Results showed that, in general, the reactions were clean and the isolated compounds were obtained, after crystallization without further purification by tedious column chromatography. It was interesting to note that the nature of functional groups on an aromatic ring in aromatic ketones has no distinct effect on the reaction time and yield as aromatic ketones possessing electron releasing as well as electron attracting groups furnished corresponding chalcones in equally good yields. Besides, heterocyclic ketones such as 3-acetyl pyridine and 2-acetyl thiophene also afforded good yield of the corresponding chalcone. The physical properties were determined and structures were confirmed by FT-IR, NMR, and HRMS spectra. The characteristic pattern of trans-coupling constant $(J=>15 \mathrm{~Hz})$ was observed in the ${ }^{1} \mathrm{H}$ NMR spectra of all compounds synthe- sized, suggesting cis isomers were not obtained. Inspection of ${ }^{1} \mathrm{H}$ NMR spectral data indicated that the compounds were geometrically pure and suggested that the $\mathrm{C}=\mathrm{C}$ in the enone linkage is in the trans-conformation.

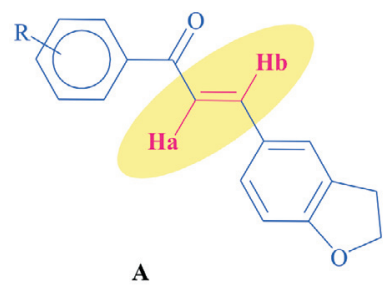

$\mathbf{A}$

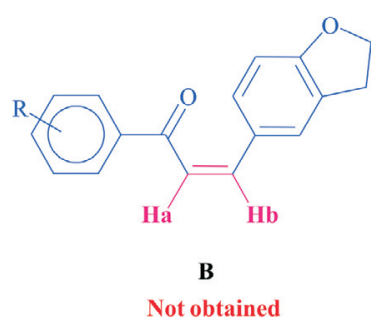

Not obtained
The probable reaction mechanism ${ }^{41}$ for the synthesis of chalcones is outlined in Scheme 2. Initially, the carbanion is generated by base induced deprotonation of acetophenone to give enolate anion (I) followed by nucleophilic attack on the carbonyl carbon of 2,3-dihydrobenzofuran-5-carbaldehyde affording $\beta$-hydroxy ketone (II) which on elimination eliminates water molecule prompting the formation of desired chalcone (III).

Table 4 Comparison between ultrasonic, stirring and reflux methods for the synthesis of $9 a^{a}$<smiles>O=Cc1ccc2c(c1)CCO2</smiles>

7<smiles>CC(=O)c1ccccc1</smiles>

$8 \mathbf{a}$<smiles>O=[N+]([O-])O</smiles>

Ethanol<smiles>O=C(/C=C/c1ccc2c(c1)CCO2)c1ccccc1</smiles>

9a

\begin{tabular}{llcc}
\hline Entry & Method & Time $/$ min & Yield $\mathrm{b} \%$ \\
\hline 1 & Reflux at $60^{\circ} \mathrm{C}$ & 120 & 45 \\
2 & Stirring at $\mathrm{rt}$ & 720 & 88 \\
3 & Ultrasound irradiation & 50 & 95 \\
\hline
\end{tabular}

${ }^{a}$ Reaction conditions:2,3-dihydrobenzofuran-5-carbaldehyde (1 mmol), acetophenone ( $1 \mathrm{mmol}), \mathrm{NaOH}$ ( $20 \mathrm{mmol}$ diluted up to $2 \mathrm{~mL}$ ).

${ }^{\mathrm{b}}$ Isolated yield of pure product. 
Table 5 Synthesis of (E)-3-(2,3-dihydrobenzofuran-5-yl)-1-(aryl)prop-2-en-1-one derivatives. ${ }^{a}$<smiles>[R]c1ccc(C(C)=O)cc1</smiles>

7
8 a to $8 n$<smiles>[R]c1ccc(C(=O)/C=C/c2ccc3c(c2)CCO3)cc1</smiles>

9a to 9n

\begin{tabular}{|c|c|c|c|c|c|}
\hline Entry & Ketone & Chalcone & Time/min & Yield $\mathrm{b} / \%$ & M.P. $/{ }^{\circ} \mathrm{C}$ \\
\hline $9 a$ & & & 50 & 95 & 108 \\
\hline $9 b$ & & & 45 & 93 & 140 \\
\hline $9 \mathrm{c}$ & & & 48 & 89 & 135 \\
\hline 9d & & & 28 & 96 & 179 \\
\hline $9 e$ & & & 22 & 88 & 105 \\
\hline 9f & & & 25 & 95 & 115 \\
\hline $9 \mathrm{~g}$ & & & 30 & 97 & 169 \\
\hline $9 \mathrm{~h}$ & & & 40 & 80 & 155 \\
\hline $9 \mathrm{i}$ & & & 45 & 85 & 159 \\
\hline 9j & & & 50 & 86 & 156 \\
\hline 9k & & & 20 & 90 & 157 \\
\hline
\end{tabular}


Table 5 (Continued).

Entry Ketone Time/min Yield $/ \%$ M.P./ ${ }^{\circ} \mathrm{C}$

${ }^{a}$ Reaction conditions: 2,3-dihydrobenzofuran-5-carbaldehyde (1 mmol), substituted acetophenone (1 mmol), NaOH (20 mmol diluted up to $\left.2 \mathrm{~mL}\right)$, ethanol (5 mL).

${ }^{\mathrm{b}}$ Isolated yield of pure product.

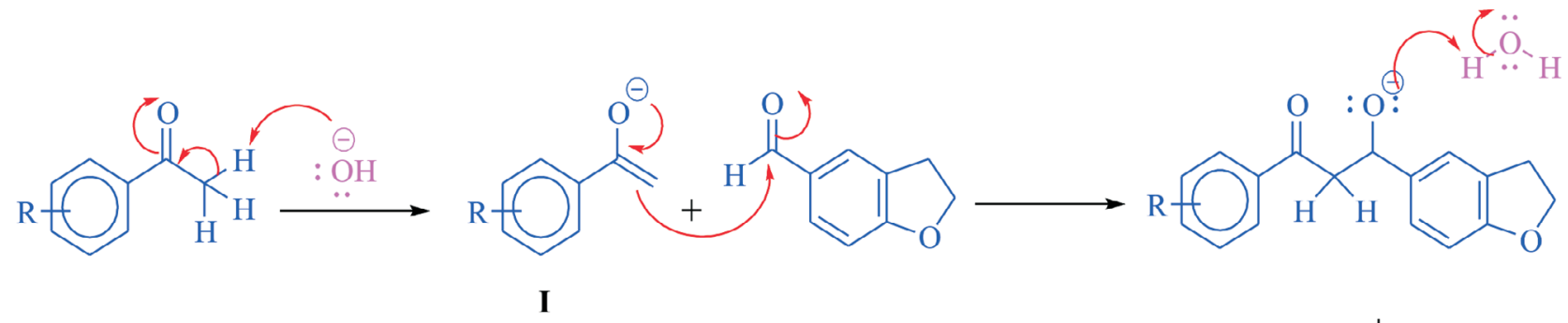<smiles></smiles>

III<smiles>[R]c1ccc(C(O)=CC(O)c2ccc3c(c2)CC2CC32CCCO)cc1</smiles>

II<smiles></smiles>

II

Scheme 2

Proposed reaction mechanism.

\section{Spectral Data of Synthesized Compounds}

9a - (E)-3-(2,3-dihydrobenzofuran-5-yl)-1-phenylprop-2-en-1one: pale yellow solid; m.p. $108^{\circ} \mathrm{C}$; FT-IR $v_{\max }\left(\mathrm{KBr} ; \mathrm{cm}^{-1}\right)$ : 2914.44, 2850.79, 1651.07, 1568.13, 1489.05, 1436.97, 1327.03, 1240.23, 1209.37, 1097.50, 1004.91; $\delta_{\mathrm{H}}(500 \mathrm{MHz}$; Choloroform-d): $8.06-7.97(\mathrm{~m}, 2 \mathrm{H}), 7.78(\mathrm{~d}, J=15.6 \mathrm{~Hz}, 1 \mathrm{H}), 7.62-7.35(\mathrm{~m}, 6 \mathrm{H}), 6.82$ $(\mathrm{d}, J=8.2 \mathrm{~Hz}, 1 \mathrm{H}), 4.65(\mathrm{t}, J=8.7 \mathrm{~Hz}, 2 \mathrm{H}), 3.26(\mathrm{t}, J=8.7 \mathrm{~Hz}, 2 \mathrm{H})$; $\delta_{\mathrm{C}}(126 \mathrm{MHz}$; Choloroform-d): 190.63, 162.65, 145.24, 138.63, 132.52, 130.16, 128.57, 128.41, 128.21, 127.79, 124.98, 119.13, 109.85, 71.96, 29.28; HRMS (ESI) calcd for $\mathrm{C}_{17} \mathrm{H}_{14} \mathrm{O}_{2}$ 251.1072, found $251.1065[\mathrm{M}+\mathrm{H}]^{+}$.

$9 b-(E)-3-(2,3-d i h y d r o b e n z o f u r a n-5-y 1)-1-(n a p h t h a l e n-2-y l)$ prop-2-en-1-one: pale yellow solid; m.p. $140{ }^{\circ} \mathrm{C}$; FT-IR $v_{\max }(\mathrm{KBr}$; $\left.\mathrm{cm}^{-1}\right)$ : 1649.14, 1581.63, 1494.83, 1427.32, 1323.17, 1286.52, 1253.73; $\delta_{\mathrm{H}}(500 \mathrm{MHz}$; Choloroform-d): $8.53(\mathrm{~d}, J=1.7 \mathrm{~Hz}, 1 \mathrm{H}), 8.10(\mathrm{dd}$, $J=8.5,1.7 \mathrm{~Hz}, 1 \mathrm{H}), 8.00(\mathrm{~d}, \mathrm{~J}=8.0 \mathrm{~Hz}, 1 \mathrm{H}), 7.94(\mathrm{~d}, J=8.6 \mathrm{~Hz}, 1 \mathrm{H})$, $7.90(\mathrm{~d}, J=8.0 \mathrm{~Hz}, 1 \mathrm{H}), 7.85(\mathrm{~d}, J=15.6 \mathrm{~Hz}, 1 \mathrm{H}), 7.64-7.52(\mathrm{~m}, 4 \mathrm{H})$, $7.48(\mathrm{dd}, J=8.3,1.9 \mathrm{~Hz}, 1 \mathrm{H}), 6.84(\mathrm{~d}, J=8.2 \mathrm{~Hz}, 1 \mathrm{H}), 4.66(\mathrm{t}, J=8.7$ $\mathrm{Hz}, 2 \mathrm{H}), 3.28(\mathrm{t}, \mathrm{J}=8.7 \mathrm{~Hz}, 2 \mathrm{H}) ; \delta_{\mathrm{C}}(126 \mathrm{MHz}$; Choloroform-d): $190.36,162.67,145.20,135.96,135.40,132.62,130.21,129.67$, $129.50,128.49,128.25,128.23,127.86,127.84,126.73,125.05$, $124.59,119.12,109.88,77.30,77.04,76.79,71.98,29.30$; HRMS (ESI) calcd for $\mathrm{C}_{21} \mathrm{H}_{16} \mathrm{O}_{2} 301.1228$, found $301.1232[\mathrm{M}+\mathrm{H}]^{+}$.

9c - (E)-3-(2,3-dihydrobenzofuran-5-yl)-1-(p-tolyl)prop-2-en1-one: pale yellow solid; m.p. $135{ }^{\circ} \mathrm{C}$; FT-IR $v \mathrm{~m}_{\mathrm{ax}}\left(\mathrm{KBr} ; \mathrm{cm}^{-1}\right)$ : 2964.59, 2912.51, 2852.72, 1653.00, 1573.91, 1492.90, 1433.11, 1330.88; $\delta_{\mathrm{H}}(500 \mathrm{MHz}$; Choloroform-d): $7.93(\mathrm{~d}, J=8.2 \mathrm{~Hz}, 2 \mathrm{H})$, $7.78(\mathrm{~d}, J=15.6 \mathrm{~Hz}, 1 \mathrm{H}), 7.53(\mathrm{~d}, J=1.9 \mathrm{~Hz}, 1 \mathrm{H}), 7.43(\mathrm{dd}, J=8.2$, $1.9 \mathrm{~Hz}, 1 \mathrm{H}), 7.40(\mathrm{~d}, J=15.6 \mathrm{~Hz}, 1 \mathrm{H}), 7.29(\mathrm{~d}, J=8.2 \mathrm{~Hz}, 2 \mathrm{H}), 6.82$ $(\mathrm{d}, J=8.2 \mathrm{~Hz}, 1 \mathrm{H}), 4.65(\mathrm{t}, J=8.7 \mathrm{~Hz}, 2 \mathrm{H}), 3.26(\mathrm{t}, J=8.7 \mathrm{~Hz}, 2 \mathrm{H})$, $2.43(\mathrm{~s}, 3 \mathrm{H}) ; \delta_{\mathrm{C}}(126 \mathrm{MHz}$; Choloroform-d): 190.09, 162.53, 144.78, $143.32,136.00,130.06,129.27,128.56,128.15,127.89,124.94$, $119.12,109.82,71.93,29.72,29.29,21.68$; HRMS (ESI) calcd for $\mathrm{C}_{18} \mathrm{H}_{16} \mathrm{O}_{2} 265.1228$, found $265.1223[\mathrm{M}+\mathrm{H}]^{+}$. 
9d - (E)-1-(2,3-dihydrobenzo[b][1,4]dioxin-6-yl)-3-(2,3dihydrobenzofuran-5-yl)prop-2-en-1-one: yellow solid; m.p. $179^{\circ} \mathrm{C}$; FT-IR $v \mu_{\text {ax }}\left(\mathrm{KBr} ; \mathrm{cm}^{-1}\right): 2912.51,2848.86,1678.07,1597.06$, $1571.99,1469.76,1435.04,1309.67,1242.16 ; \delta_{\mathrm{H}}(500 \mathrm{MHz}$; Choloroform-d): $7.76(\mathrm{~d}, J=15.5 \mathrm{~Hz}, 1 \mathrm{H}), 7.64-7.56(\mathrm{~m}, 2 \mathrm{H}), 7.52$ $(\mathrm{d}, J=1.8 \mathrm{~Hz}, 1 \mathrm{H}), 7.42(\mathrm{dd}, J=8.4,1.8 \mathrm{~Hz}, 1 \mathrm{H}), 7.36(\mathrm{~d}, J=15.5$ $\mathrm{Hz}, 1 \mathrm{H}), 6.95(\mathrm{~d}, J=8.2 \mathrm{~Hz}, 1 \mathrm{H}), 6.81(\mathrm{~d}, J=8.2 \mathrm{~Hz}, 1 \mathrm{H}), 4.64(\mathrm{t}, J=$ $8.7 \mathrm{~Hz}, 2 \mathrm{H}), 4.36-4.26(\mathrm{~m}, 4 \mathrm{H}), 3.26(\mathrm{t}, J=8.7 \mathrm{~Hz}, 2 \mathrm{H}) ; \delta_{\mathrm{C}}$ (126 MHz; Choloroform-d): 188.61, 162.48, 147.71, 144.47, 143.40, $132.35,130.02,128.13,127.94,124.89,122.53,118.78,117.96$, 117.23, 109.80, 71.92, 64.72, 64.18, 29.29; HRMS (ESI) calcd for $\mathrm{C}_{19} \mathrm{H}_{16} \mathrm{O}_{4} 309.1126$, found $309.1121[\mathrm{M}+\mathrm{H}]^{+}$.

9e - (E)-1-(2-chlorophenyl)-3-(2,3-dihydrobenzofuran-5-yl) prop-2-en-1-one: yellow solid; m.p. $105^{\circ} \mathrm{C}$; FT-IR $v_{\text {max }}\left(\mathrm{KBr} ; \mathrm{cm}^{-1}\right)$ : $3064.89,2947.23,2883.58,1653.00,1581.63,1502.55,1429.25$, 1319.31, 1282.66; $\delta_{\mathrm{H}}(500 \mathrm{MHz}$; Choloroform-d): 7.47-7.30 (m, $7 \mathrm{H}), 6.97(\mathrm{~d}, J=16.0 \mathrm{~Hz}, 1 \mathrm{H}), 6.79(\mathrm{~d}, J=8.3 \mathrm{~Hz}, 1 \mathrm{H}), 4.63(\mathrm{t}, J=8.7$ $\mathrm{Hz}, 2 \mathrm{H}), 3.23(\mathrm{t}, J=8.7 \mathrm{~Hz}, 2 \mathrm{H}) ; \delta_{\mathrm{C}}(126 \mathrm{MHz}$; Choloroform-d): $194.02,162.98,146.97,139.49,139.38,131.12,130.54,130.22$, $129.25,128.35,127.26,126.79,125.02,123.53,109.88,72.02,29.19$; HRMS (ESI) calcd for $\mathrm{C}_{17} \mathrm{H}_{13} \mathrm{ClO}_{2} 285.0682$, found 285.0678 $[\mathrm{M}+\mathrm{H}]^{+}$.

9f - (E)-1-(3-chlorophenyl)-3-(2,3-dihydrobenzofuran-5-yl) prop-2-en-1-one: pale yellow solid; m.p. $115^{\circ} \mathrm{C}$; FT-IR $v_{\max }(\mathrm{KBr}$; $\mathrm{cm}^{-1}$ ): 2960.73, 2899.01, 1643.35, 1597.06, 1573.91, 1489.05, 1436.97, 1338.60; $\delta_{\mathrm{H}}(500 \mathrm{MHz}$; Choloroform-d): $7.97(\mathrm{t}, J=1.9 \mathrm{~Hz}, 1 \mathrm{H})$, $7.88(\mathrm{dt}, J=7.9,1.5 \mathrm{~Hz}, 1 \mathrm{H}), 7.80(\mathrm{~d}, J=15.5 \mathrm{~Hz}, 1 \mathrm{H}), 7.58-7.50(\mathrm{~m}$, $2 \mathrm{H}), 7.44(\mathrm{t}, J=7.9 \mathrm{~Hz}, 2 \mathrm{H}), 7.33(\mathrm{~d}, J=15.5 \mathrm{~Hz}, 1 \mathrm{H}), 6.83(\mathrm{~d}, J=8.3$ $\mathrm{Hz}, 1 \mathrm{H}), 4.66(\mathrm{t}, J=8.7 \mathrm{~Hz}, 2 \mathrm{H}), 3.27(\mathrm{t}, J=8.7 \mathrm{~Hz}, 2 \mathrm{H}) ; \delta_{\mathrm{C}}$ (126 MHz; Choloroform-d): $\delta$ 189.15, 162.92, 146.09, 140.25, $134.83,132.42,130.44,129.90,128.49,128.31,127.53,126.46$, $125.09,118.43,109.92,77.29,77.03,76.78,72.02,29.24 ;$ HRMS (ESI) calcd for $\mathrm{C}_{17} \mathrm{H}_{13} \mathrm{ClO}_{2} 285.0682$, found $285.0678[\mathrm{M}+\mathrm{H}]^{+}$.

9g - (E)-1-(4-chlorophenyl)-3-(2,3-dihydrobenzofuran-5-yl) prop-2-en-1-one: pale yellow solid; m.p. $169^{\circ} \mathrm{C}$; FT-IR $v_{\max }(\mathrm{KBr}$; $\left.\mathrm{cm}^{-1}\right): 2902.87,2854.65,1631.78,1556.55,1487.12,1438.90,1346.31$, 1313.52, 1242.16, 1205.51; $\delta_{\mathrm{H}}(500 \mathrm{MHz}$; Choloroform-d): $7.95(\mathrm{~d}$, $J=8.5 \mathrm{~Hz}, 2 \mathrm{H}), 7.79(\mathrm{~d}, J=15.5 \mathrm{~Hz}, 1 \mathrm{H}), 7.53(\mathrm{~d}, J=1.9 \mathrm{~Hz}, 1 \mathrm{H})$, $7.47(\mathrm{~d}, J=8.5 \mathrm{~Hz}, 2 \mathrm{H}), 7.43(\mathrm{dd}, J=8.3,1.9 \mathrm{~Hz}, 1 \mathrm{H}), 7.34(\mathrm{~d}, J=$ $15.5 \mathrm{~Hz}, 1 \mathrm{H}), 6.82(\mathrm{~d}, J=8.3 \mathrm{~Hz}, 1 \mathrm{H}), 4.65(\mathrm{t}, J=8.7 \mathrm{~Hz}, 2 \mathrm{H}), 3.26(\mathrm{t}$ $J=8.7 \mathrm{~Hz}, 2 \mathrm{H}) ; \delta_{\mathrm{C}}(126 \mathrm{MHz}$; Choloroform-d $): \delta 189.20,162.83$, $145.74,138.89,136.92,130.30,129.82,128.87,128.28,127.60$, 125.06, 118.48, 109.91, 71.99, 29.25; HRMS (ESI) calcd for $\mathrm{C}_{17} \mathrm{H}_{13} \mathrm{ClO}_{2} 285.0682$, found $285.0675[\mathrm{M}+\mathrm{H}]^{+}$.

9h - (E)-3-(2,3-dihydrobenzofuran-5-yl)-1-(4-fluorophenyl) prop-2-en-1-one: yellow crystals; m.p. $155^{\circ} \mathrm{C}$; FT-IR $v_{\max }(\mathrm{KBr}$; $\left.\mathrm{cm}^{-1}\right): 2962.66,2904.80,1647.21,1568.13,1483.26,1427.32,1327.03$ $\delta_{\mathrm{H}}(500 \mathrm{MHz}$; Choloroform-d): 8.09-8.01 (m, 2H), $7.79(\mathrm{~d}, J=15.5$ $\mathrm{Hz}, 1 \mathrm{H}), 7.54(\mathrm{~d}, J=1.8 \mathrm{~Hz}, 1 \mathrm{H}), 7.44(\mathrm{dd}, J=8.2,1.8 \mathrm{~Hz}, 1 \mathrm{H}), 7.36$ $(\mathrm{d}, J=15.5 \mathrm{~Hz}, 1 \mathrm{H}), 7.17(\mathrm{t}, J=8.6 \mathrm{~Hz}, 2 \mathrm{H}), 6.83(\mathrm{~d}, J=8.2 \mathrm{~Hz}, 1 \mathrm{H})$, $4.66(\mathrm{t}, J=8.7 \mathrm{~Hz}, 2 \mathrm{H}), 3.26(\mathrm{t}, J=8.7 \mathrm{~Hz}, 2 \mathrm{H}) ; \delta_{\mathrm{C}}(126 \mathrm{MHz}$; Choloroform-d): 188.90, 162.75, 145.45, 134.93, 130.99, 130.91, 130.21, 128.24, 127.66, 125.02, 118.58, 115.74, 115.57, 109.89, 77.28, 77.03, 76.78, 71.98, 29.26; HRMS (ESI) calcd for $\mathrm{C}_{17} \mathrm{H}_{13} \mathrm{FO}_{2}$ 269.0977, found $269.0979[\mathrm{M}+\mathrm{H}]^{+}$.

9i - (E)-1-(4-bromophenyl)-3-(2,3-dihydrobenzofuran-5-yl) prop-2-en-1-one: yellow solid; m.p. $159{ }^{\circ} \mathrm{C}$; FT-IR $v_{\max }(\mathrm{KBr}$; $\left.\mathrm{cm}^{-1}\right): 2968.45,2910.58,1649.14,1579.70,1489.05,1431.18,1330.88$, $1242.16 ; \delta_{\mathrm{H}}(500 \mathrm{MHz}$; Choloroform-d): 8.09-8.01 (m, 2H), $7.79(\mathrm{~d}$, $J=15.5 \mathrm{~Hz}, 1 \mathrm{H}), 7.54(\mathrm{~d}, J=1.8 \mathrm{~Hz}, 1 \mathrm{H}), 7.44(\mathrm{dd}, J=8.2,1.8 \mathrm{~Hz}$, $1 \mathrm{H}), 7.36(\mathrm{~d}, J=15.5 \mathrm{~Hz}, 1 \mathrm{H}), 7.17(\mathrm{t}, J=8.6 \mathrm{~Hz}, 2 \mathrm{H}), 6.83(\mathrm{~d}, J=8.2$
$\mathrm{Hz}, 1 \mathrm{H}), 4.66(\mathrm{t}, J=8.7 \mathrm{~Hz}, 2 \mathrm{H}), 3.26(\mathrm{t}, J=8.7 \mathrm{~Hz}, 2 \mathrm{H}) ; \delta_{\mathrm{C}}$ (126 MHz; Choloroform-d): 189.39, 162.85, 145.81, 137.34, 131.85, $130.32,129.95,128.28,127.58,127.55,125.07,118.44,109.91,77.29$, 77.04, 76.78, 72.00, 29.24; HRMS (ESI) calcd for $\mathrm{C}_{17} \mathrm{H}_{13} \mathrm{BrO}_{2}$ 329.0177, found $329.0172[\mathrm{M}+\mathrm{H}]^{+}$.

9j-(E)-3-(2,3-dihydrobenzofuran-5-yl)-1-(3,4-dimethoxy phenyl)prop-2-en-1-one: yellow crystals; m.p. $156^{\circ} \mathrm{C}$; FT-IR $v_{\text {max }}$ $\left(\mathrm{KBr} ; \mathrm{cm}^{-1}\right): 3068.75,2966.52,2924.09,2845.00,1643.35,1568.13$, $1494.83,1444.68,1413.82,1340.53,1247.94 ; \delta_{\mathrm{H}}(500 \mathrm{MHz}$; Choloroform-d): $7.79(\mathrm{~d}, J=15.5 \mathrm{~Hz}, 1 \mathrm{H}), 7.68(\mathrm{dd}, J=8.4,2.0 \mathrm{~Hz}$, $1 \mathrm{H}), 7.62(\mathrm{~d}, J=2.0 \mathrm{~Hz}, 1 \mathrm{H}), 7.54(\mathrm{~d}, J=1.8 \mathrm{~Hz}, 1 \mathrm{H}), 7.49-7.38(\mathrm{~m}$, $2 \mathrm{H}), 6.93(\mathrm{~d}, J=8.4 \mathrm{~Hz}, 1 \mathrm{H}), 6.82(\mathrm{~d}, J=8.3 \mathrm{~Hz}, 1 \mathrm{H}), 4.65(\mathrm{t}, J=8.7$ $\mathrm{Hz}, 2 \mathrm{H}), 3.97(\mathrm{~s}, 6 \mathrm{H}), 3.26(\mathrm{t}, J=8.7 \mathrm{~Hz}, 2 \mathrm{H}) ; \delta_{\mathrm{C}}(126 \mathrm{MHz}$; Choloroform-d): 188.69, 162.48, 153.05, 149.20, 144.38, 131.70, $129.94,128.13,127.96,124.95,122.77,118.70,110.82,109.95$, $109.82,71.93,56.11,56.08,29.31$; HRMS (ESI) calcd for $\mathrm{C}_{19} \mathrm{H}_{18} \mathrm{O}_{4}$ 311.1283 , found $311.1277[\mathrm{M}+\mathrm{H}]^{+}$.

9k - (E)-3-(2,3-dihydrobenzofuran-5-yl)-1-(3-nitrophenyl) prop-2-en-1-one: brown solid; m.p. $157^{\circ} \mathrm{C}$; FT-IR $v_{\max }\left(\mathrm{KBr} ; \mathrm{cm}^{-1}\right)$ : 3095.75, 2968.45, 2908.65, 1651.07, 1566.20, 1490.97, 1438.90, $1348.24,1309.67,1246.02 ; \delta_{\mathrm{H}}(500 \mathrm{MHz}$; Choloroform-d): $8.83(\mathrm{~d}$, $J=2.0 \mathrm{~Hz}, 1 \mathrm{H}), 8.46-8.40(\mathrm{~m}, 1 \mathrm{H}), 8.35(\mathrm{~m}, 1 \mathrm{H}), 7.87(\mathrm{~d}, J=15.5$ $\mathrm{Hz}, 1 \mathrm{H}), 7.71(\mathrm{t}, J=8.0 \mathrm{~Hz}, 1 \mathrm{H}), 7.59(\mathrm{~d}, J=1.9 \mathrm{~Hz}, 1 \mathrm{H}), 7.47(\mathrm{dd}$, $J=8.3,1.9 \mathrm{~Hz}, 1 \mathrm{H}), 7.39(\mathrm{~d}, J=15.5 \mathrm{~Hz}, 1 \mathrm{H}), 6.85(\mathrm{~d}, J=8.3 \mathrm{~Hz}$, $1 \mathrm{H}), 4.68(\mathrm{t}, J=8.7 \mathrm{~Hz}, 2 \mathrm{H}), 3.29(\mathrm{t}, J=8.7 \mathrm{~Hz}, 2 \mathrm{H})$.

91 - (E)-3-(2,3-dihydrobenzofuran-5-yl)-1-(2- hydroxyphenyl) prop-2-en-1-one: yellow solid; m.p. $118^{\circ} \mathrm{C}$; FT-IR $v_{\max }\left(\mathrm{KBr} ; \mathrm{cm}^{-1}\right)$ : 3309.85, 2970.38, 2926.01, 1645.28, 1597.06, 1558.48, 1490.97, 1436.97, 1340.53; $\delta_{\mathrm{H}}(500 \mathrm{MHz}$; Choloroform-d): 12.98 (s, 1H), $7.95-7.87(\mathrm{~m}, 2 \mathrm{H}), 7.56(\mathrm{~s}, 1 \mathrm{H}), 7.52(\mathrm{~d}, J=15.4 \mathrm{~Hz}, 1 \mathrm{H}), 7.50-7.44$ $(\mathrm{m}, 2 \mathrm{H}), 7.02(\mathrm{~d}, J=8.4 \mathrm{~Hz}, 1 \mathrm{H}), 6.94(\mathrm{t}, J=7.6 \mathrm{~Hz}, 1 \mathrm{H}), 6.84(\mathrm{~d}, J=$ $8.2 \mathrm{~Hz}, 1 \mathrm{H}), 4.67(\mathrm{t}, J=8.7 \mathrm{~Hz}, 2 \mathrm{H}), 3.28(\mathrm{t}, J=8.7 \mathrm{~Hz}, 2 \mathrm{H}) . \delta_{\mathrm{C}}$ (126 MHz; Choloroform-d): 193.68, 163.56, 163.05, 145.89, 136.10, $130.59,129.50,128.36,127.54,125.26,120.18,118.73,118.61$, $116.91,109.98,72.05,29.23$.

\section{Conclusions}

In summary, the ultrasound-assisted robust protocol reported herein is characterized by short reaction times, an operationally simple method, simplicity of work-up and purification by a simple crystallization method rendering a green approach to synthesize novel (E)-3-(2,3-dihydrobenzofuran-5-yl)-1(aryl)prop-2-en-1-one chalcone derivatives. All the synthesized chalcones are novel (except 9a) with potential medicinal properties and the strategy is adequate in terms of purity and product yield. Further study is underway to investigate the biological activities of the newly synthesized compounds.

\section{Acknowledgements}

Authors are grateful to acknowledge Central Instrumentation Facility, Savitribai Phule Pune University, Pune, for NMR and mass spectral analysis. Authors are also thankful to the Lokenete Vyankatrao Hiray Arts, Science and Commerce College Panchavati, Nashik and Arts, Science and Commerce College, Surgana, for providing necessary research facilities.

\section{${ }^{5}$ ORCID iD}

V.A. Adole: (iD) orcid.org//0000-0001-7691-7884

\section{References}

1 D.N. Neubauer, A review of ramelteon in the treatment of sleep disorders, Neuropsychiatr. Dis. Treat., 2008, 4, 69-79.

2 S.R. Pandi-Perumal, V. Srinivasan, B. Poeggeler, R. Hardeland and 
D.P. Cardinali, Drug insight: the use of melatonergic agonists for the treatment of insomnia-focus on ramelteon, Nat. Clin. Pract. Neurol., 2007, 3, 221-228.

3 Z. Rozmer and P. Perjési, Naturally occurring chalcones and their biological activities, Phytochem. Rev., 2016, 15, 87-120.

4 N. Kaur and D. Kishore, Application of chalcones in heterocycles synthesis: synthesis of 2-(isoxazolo, pyrazolo and pyrimido) substituted analogues of 1,4-benzodiazepin-5-carboxamides linked through an oxyphenyl bridge, J. Chem. Sci., 2013, 125, 555-560.

5 Z.N. Siddiqui, M. Asad and S. Praveen, Synthesis and biological activity of heterocycles from chalcone, Med. Chem. Res., 2008 17, 318-325.

6 Y.H. Zaki, M.S. Al-Gendey and A.O. Abdelhamid, A facile synthesis, and antimicrobial and anticancer activities of some pyridines, thioamides, thiazole, urea, quinazoline, $\beta$-naphthyl carbamate, and pyrano[2,3-d] thiazole derivatives, Chem. Cent. J., 2018, 12, 70-83.

7 H. Rücker, N. Al-Rifai, A. Rascle, E. Gottfried, L. Brodziak-Jarosz, C. Gerhäuser, T.P. Dick and S. Amslinger, Enhancing the anti-inflammatory activity of chalcones by tuning the Michael acceptor site, Org Biomol. Chem., 2015, 13, 3040.

8 A. Kamal, M. Balakrishna, V. Reddy, S. Riyaz, C. Bagul, B.M Satyanarayana and J. Rao, Synthesis and biological evaluation of benzo[d][1,3] dioxol-5-yl chalcones as antiproliferating agents, Chem. Biol. Drug. Des., 2015, 86, 1267-1284.

9 S. Gupta, P. Maurya, A. Upadhyay, P. Kushwaha, S. Krishna, M.I Siddiqi, K.V. Sashidhara and D. Banerjee, Synthesis and bio-evaluation of indole-chalcone based benzopyrans as a promising antiligase and antiproliferative agent, Eur. J. Med. Chem., 2018, 143, 1981-1996.

10 M.Z. Gibson, M.A. Nguyen and S.K. Zingales, Design, synthesis and cytotoxic activity of certain novel chalcone analogous compounds, Med. Chem., 2018, 14, 333-343.

11 A. Kamal, M.K. Redd and A. Viswanath, The design and development of imidazothiazole-chalcone derivatives as potential anticancer drugs, Expert Opin. Drug. Discov., 2013, 8, 289-304.

12 Ü.M. Koçyiğit, Y. Budak, M.B. Gürdere, S. Tekin, T.K. Köprülü, F Ertürk, K. Özcan, I. Gülçin and M. Ceylan, Synthesis, characterization, anticancer, antimicrobial and carbonic anhydrase inhibition profiles of novel (3aR,4S,7R,7aS)-2-(4-((E)-3-(3-aryl)acryloyl) phenyl)-3a,4,7,7a-tetrahydro- $1 \mathrm{H}-4,7$-methanoisoindole-1,3(2H)dione derivatives, Bioorg. Chem., 2017, 70, 118-125.

13 N.K. Duddukuri, S. Thatikonda, C. Godugu, R.A. Kumar and N. Doijad, Synthesis of novel thiophene-chalcone derivatives as anticancer- and apoptosis-inducing agents, ChemistrySelect, 2018, 3, 6859-6864.

14 C.S. Kumar, W.S. Loh, C.W. Ooi, C.K. Quah and H.K. Fun, Structural correlation of some heterocyclic chalcone analogues and evaluation of their antioxidant potential, Molecules, 2013, 18, 11996-12011.

15 H.M. Faidallah, S.A. Rostom and K.A. Khan, Synthesis and biological evaluation of pyrazole chalcones and derived bipyrazoles as anti-inflammatory and antioxidant agents. Arch. Pharm. Res., 2015, 38, 203-215.

16 Z. Wan, D. Hu, P. Li, D. Xie and X. Gan, Synthesis, Antiviral Bioactivity of novel 4-thioquinazoline derivatives containing chalcone moiety, Molecules, 2015, 20, 11861-11874.

17 H.I. El-Subbagh, S.M. Abu-Zaid, M.A. Mahran, F.A. Badria and A.M. Al-Obaid, Synthesis and biological evaluation of certain alpha,betaunsaturated ketones and their corresponding fused pyridines as antiviral and cytotoxic agents, J. Med. Chem., 2000, 43, 2915-2921.

18 R. Pingaew, A. Saekee, P. Mandi, C. Nantasenamat, S. Prachayasittikul, S. Ruchirawat and V. Prachayasittikul, Synthesis, biological evaluation and molecular docking of novel chalconeecoumarin hybrids as anticancer and antimalarial agents, Eur. J. Med. Chem., 2014, 85, 65-76

19 N. Mateeva, S.V.K. Eyunni, K.K. Redda, U. Ononuju, T.D. Hansberry, C. Aikens and A. Nag, Functional evaluation of synthetic flavonoids and chalcones for potential antiviral and anticancer properties, Bioorg. Med. Chem. Lett., 2017, 27, 2350-2356.

20 M.N.Gomes, R.C. Braga, E.M. Grzelak, B.J. Neves, E. Muratov, R. Ma, L.L. Klein, S. Cho, G.R. Oliveira, S.G. Franzblau and C.H. Andrade, QSAR-driven design, synthesis and discovery of potent chalcone derivatives with antitubercular activity, Eur. J. Med. Chem., 2017, 137, 126-138.
21 A. Singh, C. Biot, A. Viljoen, C. Dupont, L. Kremer, K. Kumar and V. Kumar, 1H-1,2,3-Triazole-tethered uracil-ferrocene and uracil-ferrocenylchalcone conjugates: synthesis and antitubercular evaluation, Chem. Biol. Drug. Des., 2017, 89, 856-861.

22 A. Singh, A. Viljoen, L. Kremer, and V. Kumar, Synthesis and antimycobacterial evaluation of piperazyl-alkyl-ether linked 7-chloroquinoline-chalcone/ferrocenyl chalcone conjugates, ChemistrySelect, 2018, 3, 8511-8513.

23 Z.Y. Wei, K.Q. Chi, Z.K. Yu, H.Y. Liu, L.P. Sun, C.J. Zheng and H.R. Piao, Synthesis and biological evaluation of chalcone derivatives containing aminoguanidine or acylhydrazone moieties, Bioorg. Med. Chem. Lett., 2016, 26, 5920-5925.

24 T. Moodley, M. Momin, C. Mocktar, C. Kannigadu and N.A. Koorbanally, The synthesis, structural elucidation and antimicrobial activity of 2- and 4-substituted-coumarinyl chalcones, Magn. Reson. Chem., 2016, 54, 610-617.

25 U. Tutar, Ü.M. Koçyiğit and H. Gezegen, Evaluation of antimicrobial, antibiofilm and carbonic anhydrase inhibition profiles of 1,3-bis-chalcone derivatives, J. Biochem. Mol. Toxicol., 2018,33, e22281.

26 M. Kucerova-Chlupacova, J. Kunes, V. Buchta, M. Vejsova and V. Opletalova, Novel pyrazine analogs of chalcones: synthesis and evaluation of their antifungal and antimycobacterial activity, Molecules, $2015,20,1104-1117$.

27 M. Qin, H-L. Wang, J. Leng, et al. Synthesis and biological evaluation of new tetramethylpyrazine-based chalcone derivatives as potential anti-Alzheimer agents, Chem. Biol. Drug. Des., 2018, 92, 18591866.

28 G. Avila-Villarreal, O. Hernández-Abreu, S. Hidalgo-Figueroa, G. Navarrete-Vázquez, F. Escalante-Erosa, L.M. Peña-Rodríguez, R. Villalobos-Molina and S. Estrada-Soto, Antihypertensive and vasorelaxant effects of dihydrospinochalcone-A isolated from Lonchocarpus xuul Lundell by NO production: computational and ex vivo approaches, Phytomedicine, 2013, 20, 1241-1246.

29 K. Tabata, K. Motani, N. Takayanagi, R. Nishimura, S. Asami, Y. Kimura, M. Ukiya, D. Hasegawa, T. Akihisa and T. Suzuki, Xanthoangelol, a major chalcone constituent of Angelica keiskei, induces apoptosis in neuroblastoma and leukemia cells, Biol. Pharm. Bull., 2005, 28, 1404-1407.

30 C. Limper, Y. Wang, S. Ruhl, Z. Wang, Y. Lou, F. Totzke, M.H. Kubbutat, Y. Chovolou, P. Proksch and W. Watjen, Compounds isolated from Psoralea corylifolia seeds inhibit protein kinase activity and induce apoptotic cell death in mammalian cells, J. Pharm. Pharmacol., 2013, 65, 1393-1408.

31 Y. Cui, M. Ao, J. Hu and L. Yu, Anti-inflammatory activity of licochalcone A isolated from Glycyrrhiza inflata. Z. Naturforsch., C: J. Biosci., 2008, 63, 361-365.

32 M. Funakoshi-Tago, S. Tanabe, K. Tago, H. Itoh, T. Mashino, Y. Sonoda and T. Kasahara, Licochalcone A potently inhibits tumor necrosis factor alpha-induced nuclear factor-kappaB activation through the direct inhibition of IkappaB kinase complex activation, Mol. Pharmacol., 2009, 76, 745-753.

33 W. Wu, H. Ye, L. Wan, X. Han, G. Wang, J. Hu, M. Tang, X. Duan, Y Fan, S. He, et al. Millepachine, a novel chalcone, induces G2/M arrest by inhibiting CDK1 activity and causing apoptosis viamROS-mitochondrial apoptotic pathway in human hepatocarcinoma cells in vitro and in vivo, Carcinogenesis, 2013, 34, 1636-1643.

34 R.R. Kulkarni, S.G. Tupe, S.P. Gample, M.G. Chandgude, D. Sarkar, M.V. Deshpande and S.P. Joshi, Antifungal dimeric chalcone derivative kamalachalcone E from Mallotus philippinensis, Nat. Prod. Res., $2014,28,245-250$

35 C. Zhuang, W. Zhang, C. Sheng, W. Zhang, C. Xing and Z. Miao, Chalcone: a privileged structure in medicinal chemistry, Chem. Rev., 2017, 117, 7762-7810.

36 S.L. Gaonkar and U.N. Vignesh, Synthesis and pharmacological properties of chalcones: a review, Res. Chem. Intermed., 2017, 43, 6043-6077.

37 M. Draye and N. Kardos, Advances in green organic sonochemistry, Top. Curr. Chem. (Cham)., 2016, 374, 74.

38 V.A. Adole, T.B. Pawar, P.B. Koli and B.S. Jagdale, Exploration of catalytic performance of nano- $\mathrm{La}_{2} \mathrm{O}_{3}$ as an efficient catalyst for dihydropyrimidinone/thione synthesis and gas sensing, J. Nanost. Chem., $2019,9,61-76$. 
